\title{
ITERATED LOG-SCALE ORLICZ-HARDY INEQUALITIES
}

\author{
Stephen M. Buckley and Ritva Hurri-Syrjänen \\ National University of Ireland Maynooth, Department of Mathematics and Statistics \\ Maynooth, Co. Kildare, Ireland; stephen.buckley@maths.nuim.ie \\ University of Helsinki, Department of Mathematics and Statistics \\ P.O. Box 68, FI-00014 Helsinki, Finland; ritva.hurri-syrjanen@helsinki.fi
}

\begin{abstract}
We relate Orlicz-Hardy inequalities on a Euclidean domain to fatness conditions on the complement. For certain iterated log-scale distortions of $L^{n}$, this relationship is necessary and sufficient, extending results of Buckley and Koskela, and others.
\end{abstract}

\section{Introduction}

Suppose $\Omega \subset \mathbf{R}^{n}, n \geq 2$, is a bounded domain and let $d(x)=\operatorname{dist}(x, \partial \Omega)$. We consider integral Hardy inequalities

$$
\forall u \in C_{0}^{\infty}(\Omega): \quad \int_{\Omega} \Psi\left(\frac{|u(x)|}{d(x)^{1+\theta}}\right) d x \leq C \int_{\Omega} \Psi\left(\frac{|\nabla u(x)|}{d(x)^{\theta}}\right) d x,
$$

and norm Hardy inequalities

$$
\forall u \in C_{0}^{\infty}(\Omega): \quad\left\|\frac{|u(x)|}{d(x)^{1+\theta}}\right\|_{L^{\Psi}(\Omega)} \leq C\left\|\frac{|\nabla u(x)|}{d(x)^{\theta}}\right\|_{L^{\Psi}(\Omega)}
$$

where $\theta>-1$, and $\Psi:[0, \infty) \rightarrow[0, \infty)$ is in a certain class of Orlicz functions with polynomial growth; see Section 2 for a definition of the Luxemburg norm $\|\cdot\|_{L^{\Psi}(\Omega)}$. We denote by $\left(1.1_{0}\right)$ and $\left(1.2_{0}\right)$ the unweighted $(\theta=0)$ cases of $(1.1)$ and (1.2) above, which especially interest us.

For power functions $\Psi(t)=t^{p}$, it is clear that (1.1) and (1.2) are mutually equivalent. For general Orlicz functions, they may not be equivalent, but it follows easily from the subadditivity of $\Psi$ that (1.1) always implies (1.2) with a comparable constant $C$ (as noted in the unweighted case in [5, p. 790]).

As follows from [4], [16], [19], the case of Hardy inequalities for power functions on rough domains is related to a uniform $p$-fatness condition on $\Omega^{c}$; see also [14], [10], and [11]. The same is true for certain log power distortions $\Psi(t)=t^{p}\left(\log _{+}(t)\right)^{\alpha}$, where $\log _{+}(t)=\max \{1, \log t\}, t \geq 0$; see [5]. Here we consider distortions by products of powers of iterated logarithms, and relate the validity of such Hardy inequalities to fatness conditions on $\Omega^{c}$.

The Orlicz functions that most interest us are $\Psi(t)=t^{p} \psi(t)$ (especially with $p=n$ ), where $\psi(t) \in \mathrm{IL}$, IL is the class of functions $\psi=\prod_{i=1}^{k} l_{i}^{\alpha_{i}}, k \in \mathbf{N}, \alpha_{i} \in \mathbf{R}$ for each $i, l_{1}:=\log _{+}$, and $l_{i}:=l_{1} \circ l_{i-1}$ for $i>1$. For any such $\psi$, we write $e_{i}(\psi)=\alpha_{i}$ for $1 \leq i \leq k$, and $e_{i}(\psi)=0$ for $i>k$.

doi:10.5186/aasfm.2013.3837

2010 Mathematics Subject Classification: Primary 46E35.

Key words: Hardy inequality, Orlicz spaces, capacity, uniform fatness condition. 
Given $q \in \mathbf{R}$, we define the subclasses $S_{+}(q)$ and $S_{-}(q)$ of IL as follows: $\psi \in S_{+}(q)$ (or $S_{-}(q)$ ) if there exists $i \in \mathbf{N}$ such that $e_{i}(\psi)>q$ (respectively $e_{i}(\psi)<q$ ) and $e_{j}(\psi)=q$ for all $j \in \mathbf{N}, j<i$.

In the power function case $\Psi(t)=t^{p}, p>1$, a domain with uniformly $p$-fat complement satisfies $p$-Hardy; note that uniform $p$-fatness is a null condition if $p>n$. This was proved by Ancona [4] when $p=2$, and Lewis [16] and Wannebo [19] for $p \neq 2$. For Lipschitz domains, more precise results can be stated: Cianchi [7] found balance conditions between a pair of Young functions that are necessary and sufficient for the validity of norm-type Hardy inequalities. Buckley and Koskela [5, Theorem 0.4 ] then showed that domains with $p$-fat complement satisfy $\left(1.1_{0}\right)$ for $\log$ power distortions of $L^{p}$. The following result generalizes this result in two ways: it concerns the weighted case $\theta \neq 0$ and, more significantly, it involves IL-class distortions of $L^{p}$.

Theorem 1.3. Let $\Omega \subset \mathbf{R}^{n}, n>1$, be a bounded domain, and $\Psi(t)=t^{p} \psi(t)$, $1<p<\infty, \psi \in$ IL. Suppose $\mathbf{R}^{n} \backslash \Omega$ is uniformly $p$-fat. Then $\Omega$ supports the Hardy inequality (1.1) whenever $\theta>-\varepsilon$ for some $\varepsilon=\varepsilon(\psi)>0$, and with constant $C$ dependent only on $n, p, \psi, \operatorname{dia}(\Omega)$, and the fatness constants $r_{0}$ and $c$ of $\mathbf{R}^{n} \backslash \Omega$.

In the case $\Psi(t)=t^{p}$, the subcase $p=n$ is of particular importance because the Hardy inequality is then equivalent to uniform fatness of the complement [16], something that is false for $p<n$. Theorem 0.3 of [5] extended this to $L^{n}(\log L)^{\alpha}$. As a special case of our results below, we further extend this result to IL-class distortions of $L^{n}$.

Theorem 1.4. Let $\Omega \subset \mathbf{R}^{n}, n>1$, be a bounded domain, and let $\Psi(t)=t^{n} \psi(t)$, $\psi \in \mathrm{IL} \backslash S_{-}(-1)$.

(a) The unweighted integral Hardy inequality (1.10) is equivalent to the uniform $n$-fatness of $\mathbf{R}^{n} \backslash \Omega$.

(b) If $\psi \notin S_{+}(n-1)$, then the unweighted norm Hardy inequality $\left(1.2_{0}\right)$ is also equivalent to the uniform $n$-fatness of $\mathbf{R}^{n} \backslash \Omega$.

The Hardy and fatness constants in the above equivalences depend quantitatively only on each other, and on $n$, $\operatorname{dia}(\Omega)$, and $\psi$.

We will see that (1.1) is naturally associated with a uniform infimal $\Psi$-fatness condition which we show to be equivalent to uniform $p$-fatness for $\Psi(t)=t^{p} \psi(t)$, $\psi \in$ IL. By contrast, (1.2) is naturally associated with the formally weaker uniform $\Psi$-fatness condition, which is strictly weaker than its infimal variant for certain $\Psi(t)=$ $t^{p} \psi(t), \psi \in \mathrm{IL}$, at least when $p=n$.

Let $\Psi(t)=t^{n} \psi(t), \psi \in \mathrm{IL}$. By the previous paragraph, uniform infimal $\Psi$-fatness always coincides with uniform $n$-fatness. By contrast, uniform $\Psi$-fatness coincides with uniform $n$-fatness only when $\psi \notin S_{+}(n-1)$.

Theorem 1.5. Let $\Psi(t)=t^{n} \psi(t)$ for some $\psi \in$ IL. If $\psi \in S_{+}(n-1)$, then all non-empty compact sets are uniformly $\Psi$-fat. If $\psi \notin S_{+}(n-1)$, then a compact set is uniformly $\Psi$-fat if and only if it is uniformly $n$-fat.

After preliminaries in Section 2, we prove in Section 3 that a uniform infimal $\Psi$-fatness condition on the complement of a domain often implies (1.1), and we note implications in the reverse direction for Orlicz classes near $L^{n}$. In Section 4, we relate (infimal and non-infimal) $\Psi$-fatness to $p$-fatness for many Orlicz functions $\Psi$, 
including $\Psi(t)=t^{p} \psi(t), \psi \in \mathrm{IL}$, allowing us to complete the proofs of the above theorems.

We close this introduction by noting that, whenever they are true, both (1.1) and (1.2) extend by the usual limiting argument to all $u$ in $W_{0}^{1, \Psi}(\Omega)$, the $W^{1, \Psi}(\Omega)$ closure of $C_{0}^{\infty}(\Omega)$. Here, $W^{1, \Psi}(\Omega)$ is the Orlicz-Sobolev space with norm $\|u\|_{L^{\Psi}(\Omega)}+$ $\||\nabla u|\|_{L^{\Psi}(\Omega)}$, where $\|\cdot\|_{L^{\Psi}(\Omega)}$ denotes the usual Luxemburg norm on $\Omega$ with respect to $\Psi$. For more on Orlicz-Sobolev spaces, see [18], [6], and some of the references therein.

\section{Preliminaries}

In this paper, an Orlicz function is a convex homeomorphism $\Psi:[0, \infty) \rightarrow[0, \infty)$; thus Orlicz functions are a special class of Young functions. An Orlicz function $\Psi$ is well behaved if it is $C^{1}$ on $(0, \infty)$, strictly convex, and satisfies $\lim _{t \rightarrow 0^{+}} \Psi(t) / t=0$ and $\lim _{t \rightarrow \infty} \Psi(t) / t=\infty$. We single out well-behaved Orlicz functions because their conjugate functions are more easily defined than those of other Young functions, and because IL functions can be viewed as well-behaved Orlicz functions if we multiply them by suitable bounded functions (as justified later in this section). Thus it is easy to write down the conjugate function of an IL function (to within a bounded factor).

If $\Omega \subset \mathbf{R}^{n}$ is a domain and $\Psi$ an Orlicz function, the class $L^{\Psi}(\Omega)$ consists of all $f: \Omega \rightarrow \mathbf{R}$ such that $\int_{\Omega} \Psi(c|f(x)|) d x<\infty$ for some $c>0$. We define the Luxemburg norm $\|\cdot\|_{L^{\Psi}(\Omega)}$ by

$$
\|f\|_{L^{\Psi}(\Omega)}=\inf \left\{t>0 \mid \int_{\Omega} \Psi\left(\frac{|f(x)|}{t}\right) d x \leq 1\right\} .
$$

This is a norm on $L^{\Psi}(\Omega)$ once we identify functions that agree almost everywhere; see theorem III.3.2.3 in [18].

Every Orlicz function $\Psi$ has a conjugate Young function $\widetilde{\Psi}$. According to [18, p. 10], we can write $\Psi(t)=\int_{0}^{t} \psi(t) d t$ and $\widetilde{\Psi}(t)=\int_{0}^{t} \tilde{\psi}(t) d t$ for all $t>0$, where the integrands map $[0, \infty]$ to itself, are nondecreasing and left continuous, and map 0 to 0 , and where $\tilde{\psi}$ is the generalized inverse of $\psi$. For more on conjugate Young functions, see [18] and [15].

Lemma 2.2. If $\Psi$ is a well-behaved Orlicz function, then the function $\psi$ defined above is continuous and strictly increasing on $[0, \infty)$, and $\tilde{\psi}$ is its inverse. The conjugate Young function $\widetilde{\Psi}(t)=\int_{0}^{t} \tilde{\psi}(t) d t$ is also a well-behaved Orlicz function.

Proof. Convexity and $\Psi_{+}^{\prime}(0)=0$ imply that $\psi(t) \rightarrow 0$ as $t \rightarrow 0^{+}$. It follows that $\psi(0)=0$ and $\psi \in C([0, \infty))$. Strict convexity of $\Psi$ implies that $\psi$ is strictly increasing on $[0, \infty)$, and $\psi$ maps $[0, \infty)$ onto itself because of the assumption $\lim _{t \rightarrow \infty} \Psi(t) / t=$ $\infty$. Using Darboux's theorem, we deduce that $\psi$ is a self-homeomorphism of $[0, \infty)$. The fact that $\widetilde{\Psi}$ is a well-behaved Orlicz function now follows easily.

For a well-behaved Orlicz function $\Psi$, the conjugate (well-behaved) Orlicz function $\widetilde{\Psi}$ is thus given by $\widetilde{\Psi}(t)=\int_{0}^{t}\left(\Psi^{\prime}\right)^{-1}(t) d t$.

The Orlicz version of Hölder's inequality [18, p. 58] says that

$$
\left|\int_{X} f g d \mu\right| \leq 2\|f\|_{L^{\Psi}(X, \mu)}\|g\|_{L^{\tilde{\Psi}}(X, \mu)}
$$


whenever $\mu$ is a measure on $X$. In fact we have the duality relation [18, p. 61]

$$
\frac{\sup \left\{\left|\int_{X} f g d \mu\right| \mid\|g\|_{L^{\tilde{\Psi}}(X, \mu)} \leq 1\right\}}{\|f\|_{L^{\Psi}(X, \mu)}} \in[1,2] .
$$

We now discuss IL, the iterated logarithm class defined in the introduction. We define a partial order $\precsim$, and associated strict partial order $\prec$, on IL via:

$$
\begin{array}{lll}
f \precsim g & \text { if } \quad & \lim _{t \rightarrow \infty} f(t) / g(t) \leq 1, \\
f \prec g & \text { if } & \lim _{t \rightarrow \infty} f(t) / g(t)=0 .
\end{array}
$$

Let FS be the set of finite sequences, meaning sequences $\alpha=\left(\alpha_{i}\right)_{i=1}^{\infty}$ of real numbers with $\alpha_{i}=0$ for all $i>k$ for some $k$. Writing

$$
e\left(\prod_{i=1}^{k} l_{i}^{\alpha_{i}}\right)=\left(\alpha_{1}, \ldots, \alpha_{k}, 0,0, \ldots\right),
$$

we get an invertible map $e:$ IL $\rightarrow$ FS. Letting $e_{j}(\psi)$ be the $j$ th coordinate of $e(\psi)$ defines a family of maps $e_{j}: \mathrm{IL} \rightarrow \mathbf{R}, j \in \mathbf{N}$.

For $\alpha \in \mathrm{FS}$, we denote by $l^{\alpha}$ the function $\psi \in \mathrm{IL}$ with $e(\psi)=\alpha$. The degree of $\psi \in \mathrm{IL}, \operatorname{deg}(\psi)$, is the smallest value of $i$ for which $e_{i}(\psi) \neq 0$ and the co-degree, $\operatorname{codeg}(\psi)$, is the largest value of $i$ for which $e_{i}(\psi) \neq 0$. In the exceptional case $e(\psi)=0$ (equivalently, $\psi \equiv 1$ ), we write $\operatorname{deg}(\psi)=\operatorname{codeg}(\psi)=0$.

We now record some basic facts about functions in this class. The proofs via calculus and standard estimation are left to the reader. We use the notation $o(1)$ for a quantity that tends to zero as $t \rightarrow \infty$.

\section{Facts 2.5.}

(a) $\precsim$ is a total order on IL corresponding to bibliographic order on FS, i.e. $l^{\alpha} \prec l^{\beta}$ for $\alpha, \beta \in$ FS if $\alpha_{i}<\beta_{i}$ for some $i \in \mathbf{N}$, and $\alpha_{j}=\beta_{j}$ for all $j<i$.

(b) There exists $R>0$, dependent only on $\operatorname{codeg}(\psi)$, such that $t \psi^{\prime}(t)=\alpha_{i} \sigma(t)(1+$ $o(1))$ for all $t \geq R$, where $i:=\operatorname{deg}(\psi), e_{j}(\sigma)=e_{j}(\psi)-1$ for $j \leq i$, and $e_{j}(\sigma)=e_{j}(\psi)$ for $j>i$.

(c) Given $\Psi(t)=t^{p} \psi(t)$ for some $1<p<\infty$ and $\psi \in$ IL, there exists $R>0$, dependent only on $\operatorname{codeg}(\psi)$, such $\Psi^{\prime}(t)=p t^{p-1} \psi(t)(1+o(1))$ for all $t \geq R$.

(d) Given $\Psi(t)=t^{p} l^{\alpha}(t)$ for some $1<p<\infty$ and $\alpha \in$ FS, let $\Phi(t)=t^{1 / p} l^{\beta}(t)$ where $\beta \in \mathrm{FS}$ is defined by $\beta_{i}=-\alpha_{i} / p, i \in \mathbf{N}$. Then there exist $R, S>0$ such that $\Psi_{R}:=\left.\Psi\right|_{[R, \infty)}$ is strictly increasing, $\Psi_{R}(R)=S$, and the inverse function $\Psi_{R}^{-1}:[S, \infty) \rightarrow[R, \infty)$ is within a bounded factor of $\Phi_{S}:=\left.\Phi\right|_{[S, \infty)}$ (with bound dependent on $\alpha$ and $p$ ).

Proof. Part (a) follows from the fact that $\log t \prec t$. The assumption $t \geq R$ is designed to ensure that $\psi$ is differentiable; part (b) then follows easily from (a) and the elementary equation

$$
\left(l_{i}^{\alpha_{i}}\right)^{\prime}(t)=\frac{\alpha_{i} l_{i}^{\alpha_{i}-1}(t)}{t l_{1}(t) \cdots l_{i-1}(t)}
$$


Next, (c) follows from (b). As for (d), $\Psi$ is strictly increasing for large $t$ by (c). Note that $\Phi(\Psi(t)) / t$ equals $\left(l^{\alpha}(t)\right)^{1 / p} l^{\beta}(\Psi(t))$ which can be written as a product of factors of the form $\left[l_{i}(t) / l_{i}(\Psi(t))\right]^{\alpha_{i} / p}$. Since $\Psi(t) \in\left[t^{p-1}, t^{p+1}\right]$ for large $t$, we readily deduce $(d)$.

We also define the non-strict and strict bibliographic order $\precsim$ and $\prec$ on FS: $\alpha \prec \beta$ for $\alpha, \beta \in \mathrm{FS}$ if $\alpha_{i}<\beta_{i}$ for some $i \in \mathbf{N}$, and $\alpha_{j}=\beta_{j}$ for all $j<i ; \alpha \precsim \beta$ if $\alpha \prec \beta$ or $\alpha=\beta$. In view of Fact 2.5(a), these definitions are consistent with the corresponding order on IL via the identification $e:$ IL $\rightarrow$ FS.

For the remainder of this section, $\Psi(t):=t^{p} \psi(t)$, where $1<p<\infty$ and $\psi \in$ IL. In the introduction, we treated such a $\Psi$ as an Orlicz function even though $\Psi$ may fail to be increasing or convex. We can still define $\|\cdot\|_{L^{\Psi}(\Omega)}$ by $(2.1)$, but this treatment needs further justification. We show below that if we do not care about multiplication by a bounded function, we can treat $\Psi$ not just as an Orlicz function, but as a well-behaved one.

Regardless of $\psi$, we have $\Psi(t)=t^{p}$ for $0 \leq t \leq 1$, so $\Psi$ has the desired properties of a well-behaved Orlicz function on $[0,1]$. It is routine to verify that $\lim _{t \rightarrow \infty} \Psi(t) / t=$ $\infty$, and that there exists $K=K(p, \psi)>2$ such that $\Psi$ is $C^{1}$ and strictly convex on $[K, \infty), \Psi(K)>1+p K$, and $D_{K}:=\Psi^{\prime}(K)=(1+\varepsilon) p \Psi(K) / K$, for some $\varepsilon<(p-1) / 2$.

Now define $\Psi_{1}:[0, \infty) \rightarrow[0, \infty)$ to coincide with $\Psi$ on $[0,1] \cup[K, \infty)$, and by linear interpolation on $[1, K]$. Then $\Psi$ and $\Psi_{1}$ are comparable, and so $\|\cdot\|_{L^{\Psi}(\Omega)}$ and $\|\cdot\|_{L^{\Psi_{1}(\Omega)}}$ are also comparable. Since we do not care about constants depending on $p$ and $\Psi$, we ignore the distinction between $\Psi$ and $\Psi_{1}$.

$\Psi_{1}$ is an Orlicz function, but not a well-behaved one: it is not differentiable at 1 or $K$, and it fails to be strictly convex on $[1, K]$. We need to make a further adjustment to fix these shortcomings. We do this by looking at strictly increasing continuous redefinitions of $\Psi_{1}^{\prime}$ on $[1, K]$, with $\Psi_{2}^{\prime}(t)=\Psi_{1}^{\prime}(t)$ outside this interval, where $\Psi_{2}$ is subject to the continuity assumptions $\Psi_{2}^{\prime}(t)=\Psi_{1}^{\prime}(t)$ for $t \in\{1, K\}$. We need to do this in such a way that $\Psi_{2}(K)=\Psi_{1}(K)$. At one extreme, if we keep $\Psi_{2}^{\prime}(t)$ very close to $\Psi_{1}^{\prime}(1)=p$ until $t$ is very close to $K$, then $\Psi_{2}(K)$ will be very close to $p K$ which is much less than $\Psi_{1}(K)$ if $K$ is sufficiently large. At the other extreme, if $\Psi_{2}^{\prime}(t)$ is very close to $\Psi_{1}^{\prime}(K)$ already when $t$ is very close to 1 , then $\Psi_{2}(t)$ is very close to $K \Psi_{1}^{\prime}(K)$ and so strictly larger than $\Psi_{1}(K)$. An appropriate convex combination of these two extreme redefinitions of $\Psi_{1}^{\prime}$ will give $\Psi_{2}$ with the property $\int_{1}^{K} \Psi_{2}^{\prime}(t) d t=\Psi_{1}(K)-\Psi_{1}(1)$, as required.

Like $\Psi_{1}$, the function $\Psi_{2}$ is comparable to $\Psi$. Since we do not care about constants depending on $p$ and $\Psi$, we ignore the distinction between $\Psi$ and $\Psi_{2}$, and we think of $\Psi$ as being a well-behaved Orlicz function.

Remark 2.6. It follows from Lemma 2.2 and Facts 2.5(c),(d) that, modulo a bounded factor which we ignore, the conjugate Orlicz function to $\Psi(t)=t^{p} \psi(t)$ for $\psi \in \mathrm{IL}$ can be considered to be $\widetilde{\Psi}(t)=t^{p /(p-1)} \tilde{\psi}(t)$, where $e_{i}(\tilde{\psi})=-e_{i}(\psi) /(p-1)$, i.e. $\tilde{\psi}=\psi^{-1 /(p-1)}$. Of course the fudge factor involved here means that we also need to include bounded fudge factors whenever we apply (2.3) and (2.4).

We now define a sort of antiderivative of $\psi(t) / t$ which will be useful later.

Definition 2.7. For $\psi \in \mathrm{IL}$, let $I(\psi)=\phi$, where $\phi$ is the unique function in IL whose derivative is comparable to $\psi(t) / t$ for all sufficiently large $t$. Explicitly, if $i$ is 
the smallest positive integer such that $\alpha_{i} \neq-1$, then $I\left(l^{\alpha}\right)=l^{\beta}$, where $\beta_{j}=\alpha_{j}+1$ for all $j \leq i$ and $\beta_{j}=\alpha_{j}$ for $j>i$.

Finally in this section, we discuss some classes of Orlicz functions. For $C \geq 2$, define $\Delta_{2}(C)$ to consist of all Orlicz functions $\Psi$ satisfying $\Psi(2 t) \leq C \Psi(t), t>0$.

Suppose $1 \leq p<q<\infty$ and $C \geq 1$. Let $G_{p}(C)$ be the class of Orlicz functions $\Psi$ such that $\Psi\left(t^{1 / p}\right) / g(t) \in[1 / C, C], t>0$, for some convex (increasing) function $g$, let $G^{q}(C)$ be the class of Orlicz functions $\Psi$ such that $\Psi\left(t^{1 / q}\right) / h(t) \in[1 / C, C], t>0$, for some concave (increasing) function $h$, and let $G_{p}^{q}(C)=G_{p}(C) \cap G^{q}(C)$.

For any of the above classes, if the parameter $C$ is unimportant we omit it. Thus for instance $\Psi \in \Delta_{2}$ means $\Psi \in \Delta_{2}(C)$ for some $C$.

If $\Psi$ is a member of any of the Orlicz classes defined above, its growth rate is constrained. In particular, it is clear that if $\Psi \in \Delta_{2}(C)$, then $\Psi$ grows no faster than $t \mapsto t^{q}$, where $q=\log _{2} C$. Membership of $G_{p}^{q}(C)$ constrains the growth rate of $\Psi$ to be intermediate between $t \mapsto t^{p}$ and $t \mapsto t^{q}$. More precisely, a convex increasing function $f:[0, \infty) \rightarrow[0, \infty)$ which is zero only at 0 satisfies $f(s t) / f(t) \geq s$ for $t>0, s \geq 1$, and the reverse inequality is true if $f$ is concave instead of convex. We therefore deduce that

$$
\begin{array}{ll}
\forall s>1, t>0: & \Psi(s t) / \Psi(t) \geq C^{-2} s^{p}, \\
\forall s>1, t>0: & \Psi(s t) / \Psi(t) \leq C^{2} s^{q} .
\end{array}
$$

In fact (2.8) holds for $\Psi \in G_{p}(C)$ and (2.9) holds for $\Psi \in G^{q}(C)$.

For all $1 \leq p_{1}<p<p_{2}$, the function $\Psi(t)=t^{p} \psi(t), \psi \in \mathrm{IL}$, lies in $G_{p_{1}}^{p_{2}}(C)$ for some $C=C\left(p, \psi, p_{1}, p_{2}\right)$. More precisely, this follows easily from Facts $2.5(\mathrm{a}),(\mathrm{c})$, for the associated strict Orlicz function $\Psi_{2}$ defined earlier in this section, although we may need to increase the value of $K=K\left(p, p_{1}, p_{2}, \psi\right)$ used there. It is also clear that $\Psi \in \Delta_{2}(C)$ for $C=C(p, \psi)$.

Denote by $a \wedge b$ and $a \vee b$ the minimum and maximum, respectively, of numbers $a, b$.

\section{Hardy inequalities and Orlicz space fatness conditions}

In this section, we investigate the connection between Hardy inequalities and fatness conditions defined in terms of Orlicz space capacities. We recall the level- $t$ Orlicz capacities and the corresponding infimal capacities as defined in [5]; as shown there, there is a natural association between the infimal $\Psi$-capacity and (1.1), and between the level-1 $\Psi$-capacity and (1.2); see also [3], [13], and [1].

Suppose $\Psi$ is an Orlicz function and that $E$ is a compact subset of an open set $\Omega \subsetneq \mathbf{R}^{n}$. We define the level-t $\Psi$-capacity $\operatorname{cap}_{\Psi}^{t}(E ; \Omega)$ and the infimal $\Psi$-capacity $\operatorname{cap}_{\Psi}^{\inf }(E ; \Omega)$ by

$$
\begin{aligned}
\operatorname{cap}_{\Psi}^{t}(E ; \Omega) & =\inf \left\{\frac{\int_{\Omega} \Psi(|\nabla u(x)|) d x}{\Psi(t)}\left|u \in \operatorname{Lip}(\bar{\Omega}), u \geq t \chi_{E}, u\right|_{\partial \Omega}=0\right\}, \\
\operatorname{cap}_{\Psi}^{\inf }(E ; \Omega) & =\inf _{t>0} \operatorname{cap}_{\Psi}^{t}(E ; \Omega) .
\end{aligned}
$$

In particular, we write $\operatorname{cap}_{\Psi}(E ; \Omega)=\operatorname{cap}_{\Psi}^{1}(E ; \Omega)$.

Writing $A_{x, r}(y)=(y-x) / r$, a set $E \subset \mathbf{R}^{n}$ is uniformly $\Psi$-fat if there exist positive constants $r_{0}, c$, such that

$$
\forall x_{0} \in E, 0<r<r_{0}: \quad \operatorname{cap}_{\Psi}\left(\bar{B}(0,1) \cap A_{x_{0}, r}(E) ; B(0,2)\right) \geq c,
$$


and $E$ is uniformly infimally $\Psi$-fat if

$$
\forall x_{0} \in E, 0<r<r_{0}: \quad \operatorname{cap}_{\Psi}^{\inf }\left(\bar{B}(0,1) \cap A_{x_{0}, r}(E) ; B(0,2)\right) \geq c .
$$

If $E$ is uniformly $\Psi$-fat for $\Psi(t) \equiv t^{p}$, we say that $E$ is uniformly $p$-fat.

We now state a sufficient condition for (1.1) which will be a key ingredient in the proof of Theorem 1.3. Much of the proof is similar to the unweighted case proof in [5, Theorem 2.4], so we omit some details and refer to that proof.

Theorem 3.1. Suppose $\Omega \subset \mathbf{R}^{n}, n>1$, is a bounded domain, and that $1 \leq C_{0}$, $1<p<q$, and $q(n-p)<n p$. If $\mathbf{R}^{n} \backslash \Omega$ is uniformly infimally $\Psi$-fat for some Orlicz function $\Psi \in G_{p}^{q}\left(C_{0}\right)$, then there exists $\varepsilon=\varepsilon\left(p, q, C_{0}\right)>0$ such that $\Omega$ supports the integral Hardy inequality (1.1) for all $\theta \geq-\varepsilon$ with constant $C$ dependent only on $n$, $p, q$, dia $(\Omega)$, and the fatness constants $r_{0}$ and $c$ of $\mathbf{R}^{n} \backslash \Omega$.

Proof. Both (1.1) and the uniform fatness condition are scale invariant, so we may assume that $\operatorname{dia}(\Omega) \leq 1$. Suppose $u \in \operatorname{Lip}_{0}(\Omega)$, the subspace of $\operatorname{Lip}(\Omega)$ consisting of functions whose support is a compact subset of $\Omega$. For arbitrary $\alpha>0$, we derive estimates with constants of comparability dependent only on $\alpha \wedge 1$ and the parameters allowed in the statement of the theorem; any dependence on $\alpha \wedge 1$ is explicitly given.

As in the proof of [5, Theorem 2.4], we first get

$$
\int_{\Omega} \Psi\left(\frac{|u(x)|}{d^{1+\alpha}(x)}\right) d x \lesssim \frac{1}{\alpha \wedge 1} \int_{\Omega} \Psi\left(d^{-\alpha}(x)|\nabla u(x)|\right) d x .
$$

Compared with (2.13) in [5], (3.2) has the factor $\alpha \wedge 1$ in place of $\alpha$; this is because we allow $\alpha>0$ to be arbitrary, whereas $0<\alpha<1$ in [5].

(3.2) already gives the desired estimate for $\theta>0$. Suppose instead that $\theta \leq 0$. From now on we restrict $\alpha$ so that $0<\alpha<1$. Since (3.2) holds for any $\operatorname{Lip}_{0}(\Omega)$ function, we may replace $u$ by $v=u d^{\alpha-\theta}$ to deduce that

$$
\int_{\Omega} \Psi\left(\frac{|u(x)|}{d(x)^{1+\theta}}\right) d x=\int_{\Omega} \Psi\left(\frac{|v(x)|}{d^{1+\alpha}(x)}\right) d x \lesssim \frac{1}{\alpha} \int_{\Omega} \Psi\left(d^{-\alpha}(x)|\nabla v|\right) d x .
$$

But $|\nabla v| \lesssim d^{\alpha-\theta}|\nabla u|+(\alpha-\theta) d^{\alpha-\theta-1}|u|$, and so

$$
\begin{aligned}
\int_{\Omega} \Psi\left(\frac{|u|}{d^{1+\theta}}\right) d x & \leq \frac{C_{1}}{\alpha} \int_{\Omega} \Psi\left(\frac{|\nabla u|}{d^{\theta}}+(\alpha-\theta) \frac{|u|}{d^{1+\theta}}\right) d x \\
& \leq \frac{C_{2}}{\alpha}\left(\int_{\Omega} \Psi\left(\frac{|\nabla u|}{d^{\theta}}\right) d x+\int_{\Omega} \Psi\left(\frac{(\alpha-\theta)|u|}{d^{1+\theta}}\right) d x\right),
\end{aligned}
$$

where $C_{1}, C_{2} \lesssim 1$ and $C_{2}>1$.

Suppose first that $\theta=0$. Take $\alpha:=\left(2 C_{2} C_{0}^{2}\right)^{-1 /(p-1)} \approx 1$, and then $(2.8)$ implies that

$$
\frac{C_{2}}{\alpha} \int_{\Omega} \Psi\left(\frac{\alpha|u|}{d}\right) d x \leq \frac{1}{2} \int_{\Omega} \Psi\left(\frac{|u|}{d}\right) d x .
$$

From this last estimate and (3.4) we get the desired conclusion.

If instead $\theta<0$, but $-\theta \leq \alpha:=\left(2^{p} C_{2} C_{0}^{2}\right)^{-1 /(p-1)} \approx 1$, we can finish as before.

We next recall the definition in [5] of a quasilog. Given $K \geq 1$, a function $\psi:(0, \infty) \rightarrow(0, \infty)$ lies in the class $Q L(K)$ of quasilogs if $\psi(s) / \psi(t) \leq K$ whenever $s, t \in[1 / 2,2]$ or $s, t>0$ satisfy $t^{2} \wedge t^{1 / 2} \leq s \leq t^{2} \vee t^{1 / 2}$. 
Note that the opposite inequality $\psi(t) / \psi(s) \leq K$ follows whenever $s$, $t$ satisfy the same conditions, and that a quasilog is doubling in the sense that $\psi(t) / \psi(s) \leq K$ whenever $0 \leq t / 2 \leq s \leq 2 t$.

If the function $\psi$ is Lipschitz and satisfies

$$
\sup _{t>0} \int_{I_{t}} \frac{\left|\psi^{\prime}(s)\right|}{\psi(s)} d s<C<\infty,
$$

where $I_{t}$ is the interval $[1 / 2,2]$ for $1 / 2 \leq t^{2} \leq 2$, and the interval with endpoints $t$ and $t^{2}$ for all other $t>0$, then it is clear that $\psi \in Q L(\exp (C))$.

Taking $\psi$ to be any non-constant element of IL, it follows from Fact 2.5(b) that when $t$ is large, $\left|\psi^{\prime}(t)\right| / \psi(t)$ is comparable with $\phi(t)=1 /\left(t \prod_{i=1}^{j} l_{i}(t)\right)$ for some $j \in \mathbf{N}$. Since $\phi$ is the derivative of $l_{j+1}$, it follows that the left-hand side of (3.5) is finite, and so every $\psi \in$ IL is a quasilog. Conversely, it is not hard to show that quasilogs cannot grow or decay faster than bounded powers of log, with the bound dependent only on the parameter $K$. Thus, although quasilogs form a larger class than IL, the range of growth/decay rates of IL functions and quasilogs coincide.

We now recall a "Hardy implies fatness" result for Orlicz spaces near $L^{n}$.

Theorem 3.6. [5, Theorem 3.2] Let $\Omega \subset \mathbf{R}^{n}, n>1$, be a bounded domain, and $1 \leq K$. If the unweighted integral Hardy inequality (1.1 $\left.1_{0}\right)$ holds for some Orlicz function $\Psi$ such that $\Psi(t)=t^{n} \psi(t)$ for a function $\psi \in Q L(K)$ satisfying

$$
\int_{1}^{\infty} \frac{\psi(t) d t}{t}=\infty
$$

then $\mathbf{R}^{n} \backslash \Omega$ is uniformly infimally $\Psi$-fat. If instead the unweighted norm Hardy inequality (1.20) holds for such a $\Psi$, then $\mathbf{R}^{n} \backslash \Omega$ is uniformly $\Psi$-fat. In both cases, we can choose the uniform fatness constants to satisfy $r_{0}=\operatorname{dia}(\Omega)$ and $c=c(C, n, \operatorname{dia}(\Omega), \Psi)$.

It is a routine task to verify that $\psi \in$ IL satisfies $\int_{1}^{\infty}(\psi(t) / t) d t<\infty$ if and only if $\psi \in S_{-}(-1)$, thus yielding the following corollary.

Corollary 3.7. Let $\Omega \subset \mathbf{R}^{n}, n>1$, be a bounded domain, and suppose $\Psi(t)=$ $t^{n} \psi(t)$ for some $\psi \in \mathrm{IL} \backslash S_{-}(-1)$. If $\left(1.1_{0}\right)$ holds, then $\mathbf{R}^{n} \backslash \Omega$ is uniformly infimally $\Psi$-fat. If instead (1.20) holds, then $\mathbf{R}^{n} \backslash \Omega$ is uniformly $\Psi$-fat. In both cases, we can choose the uniform fatness constants to satisfy $r_{0}=\operatorname{dia}(\Omega)$ and $c=c(C, n, \operatorname{dia}(\Omega), \Psi)$.

\section{Relating Orlicz space fatness to $L^{p}$ fatness}

In this section, we relate infimal $\Psi$-fatness to $p$-fatness, and (non-infimal) $\Psi$ fatness to $n$-fatness for large classes of Orlicz functions $\Psi$, including $\Psi(t)=t^{p} \psi(t)$, $\psi \in$ IL.

Theorem 4.1. Suppose $p>1$ and suppose $\Psi \in G_{p^{\prime}} \cap \Delta_{2}$ for all $1<p^{\prime}<p$. Then every uniformly $p$-fat set is uniformly infimally $\Psi$-fat, quantitatively. If additionally there exist constants $c_{1}, c_{2} \in(0,1)$ such that $\Psi(t) / t^{p} \in\left[c_{1}, c_{1}^{-1}\right]$ for all $0<t \leq c_{2}$, then uniform $p$-fatness is quantitatively equivalent to uniform infimal $\Psi$-fatness.

We next wish to examine the connection between $n$-fatness and $\Psi$-fatness. Theorem 4.1 already gives us one direction: every uniformly $n$-fat set is uniformly $\Psi$-fat, quantitatively, as long $\Psi \in G_{p^{\prime}} \cap \Delta_{2}$ for all $1<p^{\prime}<n$. We now examine the converse. 
Theorem 4.2. The following are equivalent for $\Psi \in \Delta_{2}$ :

(a) All uniformly $\Psi$-fat sets are uniformly $n$-fat.

(b) $\operatorname{cap}_{\Psi}(\{0\} ; B(0,2))=0$.

(c) $\left\||x|^{-n+1}\right\|_{L^{\widetilde{\Psi}(B(0,2))}}=\infty$, where $\widetilde{\Psi}$ is the Orlicz dual function of $\Psi$.

Using these theorems, it is easy to prove the results stated in the introduction.

Proof of Theorem 1.3. We have $\Psi(t)=t^{p}$ for all $0 \leq t \leq c_{2}:=1$. As discussed in Section 2, $\Psi \in G_{p_{1}}^{p_{2}} \cap \Delta_{2}$ for all $1 \leq p_{1}<p<p_{2}$. The condition $p_{2}\left(n-p_{1}\right)<n p_{1}$ is satisfied when $p_{1} \in(1, p)$ and $p_{2}>p$ are both close enough to $p$. The result now follows from Theorems 3.1 and 4.1.

Proof of Theorem 1.5. By Remark 2.6, the Orlicz conjugate of $\Psi$ can be taken to be $\widetilde{\Psi}(t):=t^{n /(n-1)} \tilde{\psi}(t)$, where $\tilde{\psi}:=\psi^{-1 /(n-1)}$. Thus $\psi \in S_{+}(n-1)$ if and only if $\tilde{\psi} \in S_{-}(-1)$. It is easily verified that $\left\||x|^{-n+1}\right\|_{L^{\Phi}(B(0,2))}<\infty$ for $\Phi(t)=t^{n /(n-1)} \phi(t)$, $\phi \in \mathrm{IL}$, if and only if $\phi \in S_{-}(-1)$. The theorem now follows immediately from Theorem 4.2 and the comment preceding it.

Proof of Theorem 1.4. Theorem 1.3 implies one direction in (a) and the combination of Corollary 3.7 and Theorem 4.1 imply the converse.

As noted in the introduction, (1.10) implies $\left(1.2_{0}\right)$. Conversely if $\psi \notin S_{+}(n-1)$, then Corollary 3.7 and Theorem 1.5 together tell us that $\left(1.2_{0}\right)$ implies that $\Omega$ is uniformly $n$-fat.

We now turn to proving Theorems 4.1 and 4.2.

Proof of Theorem 4.1. Suppose $E \subset \Omega$ is a uniformly $p$-fat set, and so it is uniformly $\sigma$-fat for some $\sigma<p$, where $\sigma$ is dependent only on $p, n$, and the fatness constant [12, Theorem 1.2]. Thus there exist positive constants $r_{0}, c c$ dependent on the $p$-fatness constants, $n$, and $p$ such that

$$
\forall x_{0} \in E, 0<r<r_{0}: \quad \operatorname{cap}_{\sigma}\left(\bar{B}(0,1) \cap A_{x_{0}, r}(E) ; B(0,2)\right) \geq c .
$$

Writing $F:=A_{x_{0}, r}(E)$, we have

$$
\int_{B(0,2)}|\nabla u|^{\sigma} d x \geq c, \quad \text { whenever }\left.u\right|_{F} \equiv 1 \text { and }\left.u\right|_{\partial B(0,2)}=0 .
$$

Applying Jensen's inequality to the convex function $g$ comparable to $t \mapsto \Psi\left(t^{1 / \sigma}\right)$, we get

$$
\begin{aligned}
\left(\int_{B(0,2)} \Psi(t|\nabla u|) d x\right)^{1 / \sigma} & \approx \int_{B(0,2)} g\left(t^{\sigma}|\nabla u|^{\sigma}\right) d x \\
& \gtrsim g\left(c_{n} \int_{B(0,2)} t^{\sigma}|\nabla u|^{\sigma} d x\right) \gtrsim \Psi\left(c_{n}^{1 / \sigma} c^{1 / \sigma} t\right) \gtrsim \Psi(t),
\end{aligned}
$$

where $1 / c_{n}$ is the volume of the unit ball; the last inequality above uses the fact that $\Psi \in \Delta_{2}(C)$. It follows that $\operatorname{cap}_{\Psi}\left(\bar{B}(0,1) \cap A_{x_{0}, r}(E) ; B(0,2)\right) \geq c^{\prime}$, where $c^{\prime}$ depends on $c, n, p, q$, and the $\Delta_{2}$ and $G_{\sigma}$ parameters.

The converse is straightforward, under the assumption that $\Psi(t)=t^{p} \psi(t)$, where $\psi(t) \in\left[c_{1}, c_{1}^{-1}\right]$ for all $0 \leq t \leq c_{2}$. Suppose $F$ is a compact subset of $\bar{B}(0,1)$ satisfying $\operatorname{cap}_{\Psi}^{\inf }(F ; B(0,2)) \geq c>0$. Suppose that for a given Lipschitz function $u$ on $B(0,2)$ 
we have $\inf _{t>0} Q_{t} \geq c$, where

$$
Q_{t}:=\int_{B(0,2)}|\nabla u|^{p} \cdot \frac{\psi(t|\nabla u|)}{\psi(t)} d x .
$$

By the Fatou-Lebesgue theorem,

$$
c \leq \limsup _{t \rightarrow 0^{+}} Q_{t} \leq c_{1}^{-2} \int_{B(0,2)}|\nabla u|^{p} d x .
$$

In particular, $\operatorname{cap}_{\Psi}^{\inf }(F, B(0,2)) \leq c_{1}^{-2} \operatorname{cap}_{p}(F, B(0,2))$, and so we are done.

Proof of Theorem 4.2. Trivially (a) implies (b), since (b) just says that singleton sets are not uniformly $\Psi$-fat, and singleton sets are certainly not uniformly $n$-fat.

Suppose that (c) is false, and so $\left\||x|^{-n+1}\right\|_{L^{\widetilde{\Psi}(B(0,2))}}=A<\infty$. Applying (2.3) to the following "representation formula" (for which, see [8, Lemma 7.14])

$$
\forall u \in \operatorname{Lip}_{0}(B(0,2)): \quad|u(x)| \leq C_{n} \int_{B(0,2)} \frac{|\nabla u| d y}{|x-y|^{n-1}},
$$

we get

$$
\forall u \in \operatorname{Lip}_{0}(B(0,2)): \quad u(0) \leq 2 C_{n}\||\nabla u|\|_{L^{\Psi}(B(0,2))}\left\||x|^{-n+1}\right\|_{L^{\widetilde{\Psi}(B(0,2))}} .
$$

It follows that $\int_{B(0,2)} \Psi(|\nabla u|) \geq 1$ whenever $u \in \operatorname{Lip}_{0}(B(0,2))$ and $u(0)=2 C_{n} A$. By taking $v=u /\left(2 C_{n} A\right)$ for the set of all such functions $u$, we get the set of all test functions for $\operatorname{cap}_{\Psi}(\{0\} ; B(0,2))$. Either $2 C_{n} A \leq 1$, and we deduce that $\operatorname{cap}_{\Psi}(\{0\} ; B(0,2)) \geq 1$, or $2 C_{n} A>1$ and we deduce that $\operatorname{cap}_{\Psi}(\{0\} ; B(0,2)) \geq c$, where $c>0$ depends only on $2 C_{n} A$ and the $\Delta_{2}$ parameter. Thus we get a lower bound on $\operatorname{cap}_{\Psi}(\{0\} ; B(0,2))$ in either case, and (b) is false. By taking a contrapositive, we deduce that (b) implies (c).

Finally suppose that (c) holds. The well-known uniformly perfect condition is equivalent to $n$-fatness [9, Theorem 4.1], so a set $E$ is uniformly $n$-fat if and only if every annulus $B(x, R) \backslash B(x, r)$ contains a point of $E$ whenever $x \in E, R / r>C$, $R<\operatorname{dia}(E) / 2$, and $C$ depends on $n, \alpha$, and the fatness constant $c$. Suppose that $E$ is not uniformly $n$-fat. Thus there exists $x \in E$ and some sequence of radii $\left(R_{m}\right)$ converging to 0 such that $S_{m}:=A_{x, R_{m}}(E) \cap B(0,2) \subset B(0,1 / m), m \in \mathbf{N}$.

Let $m$ be fixed but arbitrary. Define a measure $\mu$ on the positive real axis by $d \mu(t)=c_{n} t^{n-1} d t$, where $c_{n}$ is the surface area of the unit sphere and $d t$ is Lebesgue measure. Let $\varepsilon>0$ be fixed but arbitrary. It follows from duality (2.4) that

$$
\frac{\sup \left\{\int_{\varepsilon}^{2}|f(t)| t^{1-n} d \mu(t):\|f\|_{L^{\Psi}(\mu,[\varepsilon, 2])} \leq 1\right\}}{\left\|t^{1-n}\right\|_{L^{\widetilde{\Psi}}(\mu,[\varepsilon, 2])}} \in[1,2] .
$$

By positivity of $|t|^{1-n}$ and the Dominated Convergence Theorem, we may restrict the above supremum to positive bounded functions. Writing $A_{\varepsilon}:=\left\|t^{1-n}\right\|_{L^{\widetilde{\Psi}(\mu,[\varepsilon, 2])}}$, there thus exists a positive bounded $f_{\varepsilon}$ on $[\varepsilon, 2]$ with

$$
\left\|f_{\varepsilon}\right\|_{L^{\Psi}(\mu,[\varepsilon, 2])} \leq 1 / A_{\varepsilon}
$$

and

$$
\int_{\varepsilon}^{2} f_{\varepsilon}(t) t^{1-n} d \mu(t) \in[1 / 2,2]
$$


It follows immediately that by multiplying $f_{\varepsilon}$ by a suitable constant between $c_{n} / 2$ and $2 c_{n}$, we get a function $g_{\varepsilon}$ on $[\varepsilon, 2]$ such that $\left\|g_{\varepsilon}\right\|_{L^{\Psi}(\mu,[\varepsilon, 2])} \leq 2 c_{n} / A_{\varepsilon}$ and

$$
\int_{\varepsilon}^{2} g_{\varepsilon}(t) d t=\int_{\varepsilon}^{2} c_{n}^{-1} g_{\varepsilon}(t) t^{1-n} d \mu(t)=1
$$

Now letting

$$
u_{\varepsilon}(x)= \begin{cases}1, & |x| \leq \varepsilon \\ 1-\int_{\varepsilon}^{|x|} g_{\varepsilon}(x) d t, & \varepsilon \leq|x| \leq 2\end{cases}
$$

we get an admissable function for the capacity of the annulus $\bar{B}(0,2) \backslash B(0, \varepsilon)$ such that $\left\|\nabla u_{\varepsilon}\right\|_{L^{\Psi}(B(0,2))} \leq 2 c_{n} / A_{\varepsilon}$. Since this upper bound tends to zero as $\varepsilon \rightarrow 0^{+}$, and $\Psi(s t) \geq s \Psi(t)$ for all $s \geq 1$ and $t>0$ (by convexity), it follows that the $\operatorname{cap}_{\Psi}(\bar{B}(0,2) \backslash B(0, \varepsilon)) \rightarrow 0$ as $\varepsilon \rightarrow 0$. Applying this result to $S_{m}, m \in \mathbf{N}$, we deduce that any set $E$ that fails to be uniformly $n$-fat also fails to be uniformly $\Psi$-fat. Taking the contrapositive, we get (a), as required.

Finally we consider the relationship between the capacities associated with $\Psi(t)=$ $t^{n} \psi(t)$ and certain associated Hausdorff contents. This will in many cases allow us to give an associated Cantor set $E$ such that $E$ is a null set for $\psi:=\psi_{1}$ but not for $\psi:=\psi_{2}$, where $\psi_{1} \prec \psi_{2}$, illustrating how many of these capacities are pairwise distinct, in contrast to the associated uniform fatness conditions which, as we have seen, are pairwise equivalent when $\psi \notin S_{+}(n-1)$ (and separately pairwise equivalent when $\left.\psi \in S_{+}(n-1)\right)$.

Denote by $\mathcal{H}_{r}^{h}(E)$ and $\mathcal{H}^{h}(E)$ the Hausdorff content and Hausdorff measure, respectively, of $E \subset \mathbf{R}^{n}$ with respect to a (continuous increasing) gauge function $h:[0, \infty) \rightarrow[0, \infty)$. For the basic theory of Hausdorff contents and measures, see [17] or [2].

Below we are interested only in IL-class gauge functions, i.e. those of the form $h(t)=\phi(1 / t), \phi \in$ IL. Since a gauge function $h$ is required to satisfy $h(t) \rightarrow 0$ as $t \rightarrow 0^{+}$, such a $\phi$ must be of positive degree and satisfy $e_{i}(\phi)<0$ for $i:=\operatorname{deg}(\phi)$. Given this assumption, it is clear that we can multiply $\phi$ by a bounded function so as to ensure that $h$ is increasing (and continuous). As usual, we gloss over this minor adjustment of $\phi$, so when we say that $h(t):=\phi(1 / t), t \in \mathrm{IL}$, is a gauge function we simply mean that $e_{i}(\phi)<0$ for $i:=\operatorname{deg}(\phi)$.

Theorem 4.4. Suppose $\Psi(t)=t^{n} \psi(t)$ for $\psi=l^{\alpha} \in \mathrm{IL} \backslash S_{-}(-1)$, and $k \in \mathbf{N}$, $k \geq \operatorname{codeg}(\psi)$. Let $E$ be an arbitrary compact subset of $B:=B(0,1)$, and let $E+t:=\left\{x \in \mathbf{R}^{n}: \operatorname{dist}(x, E) \leq t\right\}, t>0$.

(a) If $h(t)=\phi(1 / t) \cdot l_{1}^{-n}(1 / t)$ is a gauge function, where $\phi:=I(\psi)$ is as in Definition 2.7, then there exists $C=C(n, \alpha)$ such that $\operatorname{cap}_{\Psi}(E ; 2 B) \leq C \mathcal{H}_{\infty}^{h}(E)$.

(b) Suppose $h(t)=l^{\beta}(1 / t)$ is a gauge function, for some $\beta \in$ FS such that $\beta_{i}=$ $\alpha_{i}+1-n, i<k$, and $\beta_{k}<\alpha_{k}+1-n$. If $|E+t| \leq C_{0} t^{n} / h(t)$ for all $0<t<1$, and if $\mathcal{H}_{\infty}^{h}(E)>c_{0}>0$, then there exists $c=c\left(n, \alpha, \beta, c_{0}, C_{0}\right)>0$ such that $\operatorname{cap}_{\Psi}(E ; B(0,2))>c$. 
Proof. We first prove (a). Let $|x|<5 / 4$ and $r \leq 1 / 4$. By taking

$$
u(y)= \begin{cases}1, & |y-x| \leq r, \\ (\log (1 / 2 r))^{-1} \log (1 / 2|y-x|), & r<|y-x|<1 / 2, \\ 0, & |y-x| \geq 1 / 2,\end{cases}
$$

it follows that

$$
\int_{2 B} \Psi(|\nabla(u)|) d y=\int_{r<|y|<1 / 2}|x|^{-n} \log ^{-n}(1 / 2 r) \cdot \psi\left(\frac{1}{|y| \log (1 / 2 r)}\right) d y .
$$

By two more changes of variables, we get

$$
\begin{aligned}
l_{1}^{n}(1 / r) \int_{2 B} \Psi(|\nabla(u)|) d y & \approx \int_{r}^{1 / 2} \psi\left(\frac{1}{t l_{1}(1 / r)}\right) \frac{d t}{t} \\
& \left.=\int_{2 / l_{1}(1 / r)}^{1 / r l_{1}(1 / r)} \frac{\psi(s) d s}{s} \approx \phi(s)\right]_{2 / l_{1}(1 / r)}^{1 / r l_{1}(1 / r)} \approx \phi(1 / r) .
\end{aligned}
$$

The proof of (a) now follows as usual from the subadditivity of cap ${ }_{\Psi}$. The restrictions on $x$ and $r$ in the capacity estimate are not a problem: since trivially $\left.\operatorname{cap}_{\Psi}(E ; 2 B)\right) \lesssim 1$, we may without loss of generality assume that $\mathcal{H}_{\infty}^{h}(E)<c$, where $c=c_{\alpha, n}>0$ is chosen so that any associated $\mathcal{H}_{\infty}^{h}$-efficient covering of $E$ must use balls of radius at most $1 / 4$.

We now prove (b). By Frostman's lemma [2, p.6], there is a Radon measure $\mu$ supported on $E$ such that $\mu(B(x, r)) \leq h(r)$ for all $x \in \mathbf{R}^{n}$, and $\mu(E) \geq a c_{0}$ for some $a=a(n)>0$. It follows from (4.3) that if $u \in \operatorname{Lip}_{0}(B(0,2))$ and $u \geq 1$ on $E$, then

$$
\begin{aligned}
1 \lesssim \mu(E) & \leq \int_{E} \int_{B(0,2)} \frac{|\nabla u(y)| d y}{|x-y|^{n-1}} d \mu(x) \\
& =\int_{B(0,2)}|\nabla u(y)| \int_{E} \frac{d \mu(x)}{|x-y|^{n-1}} d y \lesssim \int_{B(0,2)}|\nabla u(y)| \cdot \frac{h(\delta(y))}{\delta(y)^{n-1}} d y,
\end{aligned}
$$

where $\delta(y)=\operatorname{dist}(y, E)$. Applying Hölder's inequality (2.3), we see that

$$
1 \lesssim\||\nabla u|\|_{L^{\Psi}(B(0,2))}\left\|\frac{h(\delta(y))}{\delta(y)^{n-1}}\right\|_{L^{\tilde{\Psi}(B(0,2))}}
$$

where $\widetilde{\Psi}(t) \approx t^{n /(n-1)} /(\psi(t))^{1 /(n-1)}$, by Remark 2.6. A little calculation gives

$$
\int_{B(0,2)} \widetilde{\Psi}\left(\frac{h(\delta(y))}{\delta(y)^{n-1}}\right) d y \approx \int_{B(0,2)} \frac{h(\delta(y))}{\delta(y)^{n} \eta(1 / \delta(y))} d y
$$

where $\eta \in \mathrm{IL}, \eta=l^{\gamma}, \gamma_{i}=1$ for $i<k$, and $\gamma_{k}=1+\varepsilon$ with $\varepsilon=(\alpha-\beta) /(n-1)-1>0$. Defining $A_{m}=\left(E+2^{-m+2}\right) \backslash\left(E+2^{-m+1}\right), m \in \mathbf{N}$, we see that

$$
\int_{A_{m}} \frac{h(\delta(y))}{\delta(y)^{n} \eta(1 / \delta(y))} d y \lesssim \frac{1}{m\left(\prod_{i=1}^{k-1} l_{i}(m)\right) \cdot l_{k-1}^{\varepsilon}(m)} .
$$

The decay rate of $|E+t|$ implies that $|E|=0$, so by summation above we get

$$
\int_{B(0,2)} \frac{h(\delta(y))}{\delta(y)^{n} \eta(1 / \delta(y))} d y \lesssim 1
$$

and so the rightmost norm in (4.5) is bounded. Thus $1 \lesssim\||\nabla u|\|_{L^{\Psi_{\beta}(B(0,2))}}$, and we get the desired capacity lower bound. 
In the above proof, the assumption $\psi \notin S_{-}(-1)$ is needed only in (a), to ensure that the antiderivative of $s \mapsto \psi(s) / s$ is comparable to a positive multiple of $I(\psi)$.

In the generic case $\alpha_{1} \neq-1$, we have $h(t)=l^{\beta}(1 / t)$ in Theorem 4.4(a), where $\beta_{1}=\alpha_{1}+1-n$, and $\beta_{i}=\alpha_{i}, i>1$. The gap between this formula for $\beta$ and that in Part (b) (a gap that is a little larger when $\alpha_{1}=-1$ ) means that Theorem 4.4 is not strong enough to separate arbitrary pairs of capacities of the form $\Psi(t)=t^{n} \psi(t)$, $\psi \in \mathrm{IL}$, but we now examine what pairs it does separate.

Choosing a gauge function $h_{\beta}(t):=l^{\beta}(1 / t), \beta \in \mathrm{FS}$, it follows as in [17, 4.11] that there exists a Cantor set $F_{\beta} \subset[0,1]$ such that $1 / 4 \leq \mathcal{H}^{h_{\beta}}\left(F_{\beta}\right) \leq 1$, and such that the $k$ th approximation to $F_{\beta}$ consists of $2^{k}$ intervals each of length $s_{k}$, where $h_{\beta}\left(s_{k}\right)=2^{-k}$. Thus $\mathcal{H}^{h_{\gamma}}\left(F_{\beta}\right)=0$ for all $\gamma \prec \beta$ and $\mathcal{H}^{h_{\gamma}}\left(F_{\beta} \cap \bar{B}(x, r)\right)=\infty$ whenever $x \in F_{\beta}, r>0, \beta \prec \gamma$, and $h_{\gamma}$ is a gauge function. Letting $E_{\beta}=J\left(F_{\beta}\right)$, where $J$ is the usual identification of the real line with the first coordinate axis in $\mathbf{R}^{n}$, we see that $E_{\beta}$ inherits the above properties of $F_{\beta}$. Additionally $\left|E_{\beta}+t\right| \lesssim(3 t)^{n} / h_{\beta}(t)$, since $E_{\beta}+t$ can be covered by at most $1 / h_{\beta}(t)$ cubes of sidelength $3 t$.

Now let $\Psi(t)=t^{n} l^{\alpha}(t)$. If $I(\psi) l_{1}^{-n}=l^{\gamma}$ for some $\gamma \prec \beta$ then $\mathcal{H}^{h_{\gamma}}\left(E_{\beta}\right)=0$ and

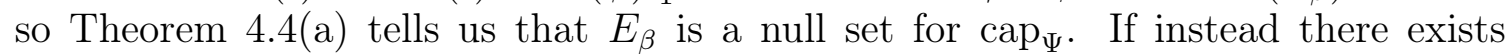
$j \in \mathbf{N}$ such that $\alpha_{i}=\beta_{i}+n-1$ for $i<j$, and $\alpha_{j}>\beta_{j}+n-1$, and we define $\gamma \in \mathrm{FS}$ by $\gamma_{i}:=\beta_{j}$ for $i<j, \beta_{j}<\gamma_{j}<\alpha_{j}-n+1$, and $\gamma_{i}:=0$ for $i>j$, then $\mathcal{H}^{h_{\gamma}}\left(E_{\beta} \cap \bar{B}(x, r)\right)=\infty$ for all $x \in E_{\beta}, r>0$. This estimate plus Theorem 4.4(b) together imply that $E_{\beta}$ is not a null set for $\operatorname{cap}_{\Psi}$.

The calculations in the above paragraph yield the following corollary; the assumption $\beta_{1}<n-1$ is made for simplicity but could be relaxed a little.

Corollary 4.6. Suppose $\Phi(t)=t^{n} l^{\alpha}(t)$ and $\Psi(t)=t^{n} l^{\beta}(t)$, where $\alpha \prec \beta$ and $\beta_{1}<n-1$. If $\alpha_{1}<\beta_{1}$, or $\alpha_{1}=\beta_{1} \neq-1$ and $\alpha_{2}<\beta_{2}+1-n$, or $\alpha_{1}=\beta_{1}=-1$ and $\alpha_{2}<\beta_{2}-n$, then there exists a Cantor set in $\mathbf{R}^{n}$ which is a null set for cap p $_{\Phi}$ but not for $\operatorname{cap}_{\Psi}$.

Cantor sets sometimes distinguish between $\Psi$-capacity and infimal $\Psi$-capacity.

Corollary 4.7. Suppose $\Psi(t)=t^{n} l^{\beta}(t)$, where $\beta_{1}<n-1$. Suppose further that either $\beta_{1}>0$, or $\beta_{1}=0$ and $\beta_{2}>n-1$. Then there exists a Cantor set in $\mathbf{R}^{n}$ which is a null set for cap inf but not for $\operatorname{cap}_{\Psi}$.

Proof. By the previous corollary with $\Phi(t)=t^{n}$, there exists a Cantor set in $\mathbf{R}^{n}$ which is a null set for $\operatorname{cap}_{\Phi}$ but not for $\operatorname{cap}_{\Psi}$. By contrast, the last line in the proof of Theorem 4.1 tells us that infimal $\Psi_{0}$-capacity is always dominated by $n$-capacity for $\Psi_{0}=\Psi$, or indeed for any $\Psi_{0}(t)=t^{n} \psi_{0}(t), \psi_{0} \in$ IL.

\section{References}

[1] Adams, D. R., and R. Hurri-Syrjänen: Capacity estimates. - Proc. Amer. Math. Soc. 131:4, 2003, 1159-1167.

[2] Aikawa, H., and M. Essén: Potential theory — selected topics. - Lecture Notes in Math. 1633, Springer-Verlag, Berlin, 1996.

[3] Aïssaoui, N., and A. Benkirane: Capacités dans les espaces d'Orlicz. - Ann. Sci. Math. Québec 18:1, 1994, 1-23.

[4] Ancona, A.: On strong barriers and an inequality of Hardy for domains in $\mathbf{R}^{n}$. - J. London Math. Soc. (2) 34:2, 1986, 274-290. 
[5] Buckley, S. M., and P. Koskela: Orlicz-Hardy inequalities. - Illinois J. Math. 48:3, 2004, $787-802$.

[6] Cianchi, A.: A sharp imbedding theorem for Orlicz-Sobolev spaces. - Indiana Univ. Math. J. 45:1, 1996, 39-65.

[7] Cinnchi, A.: Hardy inequalities in Sobolev spaces. - Trans. Amer. Math. Soc. 351:6, 1999, 2459-2478.

[8] Gilbarg, D., and N.S. Trudinger: Elliptic partial differential equations of second order, second edition. - Springer-Verlag, Berlin, 1983.

[9] JÄRVI, P., and M. Vuorinen: Uniformly perfect sets and quasiregular mappings. - J. London Math. Soc. (2) 54:3, 1996, 515-529.

[10] Korte, R., and N. Shanmugalingam: Equivalence and self-improvement of $p$-fatness and Hardy's inequalities, and association with uniform perfectness. - Math. Z. 264:1, 2010, 99-110.

[11] Korte, R., J. Lehrbäck, and H. Tuominen: The equivalence between pointwise Hardy inequalities and uniform fatness. - Math. Ann. 351:3, 2011, 711-731.

[12] Koskela, P., and X. Zhong: Hardy's inequality and the boundary size. - Proc. Amer. Math. Soc. 131:4, 2002, 1151-1158.

[13] Kuznetsov, S. E.: Removable singularities for $L u=\psi(u)$ and Orlicz capacities. - J. Funct. Anal. 170:2, 2000, 428-449.

[14] Lenrbäck, J.: Pointwise Hardy inequalities and uniformly fat sets. - Proc. Amer. Math. Soc. $136: 6,2008,2193-2200$.

[15] LÉonard, C.: Orlicz spaces. - Preprint, available at http://www.cmap.polytechnique.fr/ $\sim$ leonard/papers/orlicz.pdf.

[16] Lewis, J. L.: Uniformly fat sets. - Trans. Amer. Math. Soc. 308:1, 1988, 177-196.

[17] Mattila, P.: Geometry of sets and measures in Euclidean spaces. - Cambridge Univ. Press, Cambridge, 1995.

[18] Rao, M. M., and Z. D. Ren: Theory of Orlicz spaces. - Marcel Dekker, New York, 1991.

[19] Wannebo, A.: Hardy inequalities. - Proc. Amer. Math. Soc. 109:1, 1990, 85-95.

Received 31 October $2012 \bullet$ Accepted 11 January 2013 